\title{
Hypothesis
}

\section{COVID-19 Pandemic: How to Use Artificial Intelligence to Choose Non- Vulnerable Workers for Positions with the Highest Possible Levels of Exposure to the Novel Coronavirus}

${ }^{1} \mathrm{MD}$, School of Medicine, Shiraz University of Medical Sciences,

Shiraz, Iran

${ }^{2} \mathrm{PhD}$, Department of Medical Physics and Engineering, School of Medicine, Shiraz University of Medical Sciences, Shiraz, Iran

Mortazavi S. A. R. ${ }^{1 \oplus}$, Mortazavi S. M. J.2, Parsaei H. ${ }^{2 * \odot}$

\begin{abstract}
The COVID-19 pandemic has affected people globally; nowadays several countries are facing a major change in daily life due to universal quarantining, closed schools, social isolation, and shelter-in-place orders. In addition, this pandemic caused an economic crisis. International Labour Organization (ILO) reported that not only COVID-19 is a serious threat to public health but also the economic and social disruption of this crisis threatens the long-term livelihoods and wellbeing of millions of people. In this short paper, we introduce our hypothesis on using artificial intelligence (AI) technology to predict which employees are most vulnerable to the infections caused by the novel coronavirus (SARS-CoV-2). Such a system can be used in a wide variety of work places such as libraries, banks, drugstores, and hotel receptions to reduce the risk of severe infections in employees as well as to ensure the safety of labor force and the sustainability of businesses and jobs.
\end{abstract}

Citation: Mortazavi SAR, Mortazavi SMJ, Parsaei H. COVID-19 Pandemic: How to Use Artificial Intelligence to Choose Non-Vulnerable Workers for Positions with the Highest Possible Levels of Exposure to the Novel Coronavirus. J Biomed Phys Eng. 2020;10(3):383-386 doi: $10.31661 / \mathrm{jbpe.v010.2004-1106.}$

Keywords

COVID-19; Coronavirus; Machine Learning; Prediction Model; Classification

\section{Introduction}

T he Coronavirus disease 2019 (COVID-19) was first detected in early December 2019 in Wuhan, China [1]. This pandemic has affected people globally, regardless of their nations, races, and socioeconomic groups. Many countries now are facing a major change in daily life due to universal quarantining, closed schools, social isolation, and shelter-in-place orders [2]. The novel coronavirus (SARS-CoV-2) can be transmitted by respiratory droplets and physical contact. The incubation period for SARS-CoV-2 is around 3 to 7 days, but in some cases, this period can be as long as 24 days [3]. COVID-19 had quickly spread to
*Corresponding author: H. Parsaie

Department of Medical

Physics and Engineering

School of Medicine, Shi-

raz University of Medical

Sciences, Shiraz, Iran

E-mail: hparsaei@sums. ac.ir

Received: 18 April 2020 Accepted: 4 May 2020 
different countries and as of April 6, 2020, it caused more than 60000 deaths [4]. As of April 11, 2020, more than 1.6 million COVID-19 cases are reported globally. Scientists believe that currently it is not clear how or when COVID-19 tragedy will end. Data collected from 21 hospitals in Wuhan, China shows that among 168 patients who died, $75 \%$ were male. While the median age was 70 years, about $96 \%$ were older than 50 years. Moreover, about 74 had 1 or more comorbidities. The most common comorbidities were hypertension (50\%), diabetes $(25 \%)$, and ischemic heart disease (18.5\%)[4]. Moreover, in Italy, among 1591 laboratoryconfirmed COVID-19 patients admitted to ICUs of the Lombardy Region, the median age was 63 years and $82 \%$ were male. Furthermore, among 1043 patients with available data, $68 \%$ had at least one comorbidity (hypertension 49\%) [5].

\section{Economic Crisis}

International Labour Organization (ILO) has announced recently that about 25 million jobs could be lost globally as a result of COVID-19 [6]. Given this consideration, COVID-19 not only created a health care crisis but an economic crisis. About 10 million people filed for unemployment insurance during the 2 weeks ending on March 28, 2020. Moreover, it has been suggested that the US economy will contract by $10-25 \%$ during the second quarter. Now, there is a growing global concern over the economic costs of throwing a very large proportion of people out of work. A long-term lockdown can trigger a sharp rise in unemployment and hence, more domestic violence. ILO believes that not only COVID-19 is a serious threat to public health, but the economic and social disruption of this crisis threatens the long-term livelihoods and wellbeing of millions of people.

\section{AI Against COVID-19}

Recent reports indicate that Artificial Intelligence (AI) can be introduced as a potentially powerful tool in the global fight against the COVID- 19 pandemic [7-10]. AI has also been introduced as an effective tool to accurately predict which newly infected patients will develop the severe respiratory disease (ARDS). Considering the growing capabilities of AI, we believe that AI techniques might help employers choose non-vulnerable workers for positions with the highest possible levels of exposure to the virus. In this short paper, we discuss how a local COVID-19 hospitalization/mortality database in each country can be used to estimate hospitalization and mortality risk for each worker. Therefore, in a library, a bank or a store, non-vulnerable employees can be selected for responsibilities with the highest probability of exposure to the virus.

Developing an AI model, in general, includes three steps: data collection, feature extraction/selection, and model development. Hospitalization/mortality database in each country or worldwide with laboratory-confirmed COVID-19 can be used as the database. In terms of features, we hypothesize that features from demographic, socio-economic and lifestyle factors, patient's health status, physiological data could be used as inputs of the AI-based model. Particularly, we propose using features such as gender, age, general medical 
information such as comorbidities (diabetes, cardiovascular disease, respiratory disease, iron deficiency anemia), number of services, number of COVID-19 cases are reported in the vacancy of the office.

For feature selection and dimensionality reduction, well-known feature selection methods including filter method (univariate and multivariate) and wrapper method (forward selection, backward elimination or floating search methods) could be used [11]. The objective of this step is to remove irrelevant variables and select a subset of variables (predictors) for use in the predictive model construction. Feature selection step should be used to simplify the model, fasten the training process, avoid the curse of dimensionality, improve generalization (model's ability to predict the output for new data), and to make the model easier to interpret and understand by the users.

In terms of the model, several machine learning algorithms such as decision trees, support vector machines, Neural Networks, Random Forest, or Deep learning could be used as these models were successful tools in predicting medical conditions and adverse events even in the Patients with COVID-19 [6, 7, 9].

In short, it this letter we discussed how AI capabilities can assist employers/managers to decide which employers most vulnerable to the virus and finally manage them. We believe that this AI-based model might be used in a wide variety of work places ranging from libraries, to banks, drug stores, and hotel receptions to reduce the risk of severe infections in employees as well as to ensure the safety of labor force and the sustainability of busi- nesses and jobs.

\section{Conflict of Interest}

None declared by the authors

\section{References}

1. Pan A, Liu L, Wang C, Guo H, Hao X, Wang Q, Huang J, He N, Yu H, Lin X, Wei S. Association of public health interventions with the epidemiology of the COVID-19 outbreak in Wuhan, China. JAMA. 2020. doi: 10.1001/ jama.2020.6130.

2. Shanafelt T, Ripp J, Trockel M. Understanding and addressing sources of anxiety among health care professionals during the COVID-19 pandemic. JAMA. 2020.

3. Yang $Y$, Lu Q, Liu $M$, Wang $Y$, Zhang $A$, Jalali N, Dean N, Longini I, Halloran ME, Xu B, Zhang X. Epidemiological and clinical features of the 2019 novel coronavirus outbreak in China. MedRxiv. 2020. doi: 10.1101/2020.02.10.20021675.

4. Wang D, Hu B, Hu C, Zhu F, Liu X, Zhang $J$, Wang $B$, Xiang $H$, Cheng $Z$, Xiong $Y$, Zhao Y. Clinical characteristics of 138 hospitalized patients with 2019 novel coronavirus-infected pneumonia in Wuhan, China. JAMA. 2020;323(11):1061-9. doi: 10.1001/ jama.2020.1585. PubMed PMID: 32031570. PubMed PMCID: PMC7042881.

5. Grasselli G, Zangrillo A, Zanella A, Antonelli M, Cabrini L, Castelli A, Cereda D, Coluccello A, Foti G, Fumagalli R, Iotti G. Baseline characteristics and outcomes of 1591 patients infected with SARS-CoV-2 admitted to ICUs of the Lombardy region, Italy. JAMA. 2020;323(16):1574-1581. doi: 10.1001/ jama.2020.5394.

6. International Labour Organization. COVID-19: Protecting workers in the workplace: Almost 25 million jobs could be lost worldwide as a result of COVID-19. ILO; 2020.

7. Wynants L, Van Calster B, Bonten MMJ, Collins GS, Debray TP, De Vos M, Haller MC, Heinze G, Moons KG, Riley RD, Schuit E. Prediction models for diagnosis and prognosis of covid-19 infection: systematic review and critical appraisal. BMJ. 2020;369. 
Mortazavi. S. A. R., Mortazavi S. M. J., Parsaei H.

8. Wim N. Artificial Intelligence against COVID-19: An Early Review. IZA DP No. 13110; Germany: IZA - Institute of Labor Economics; 2020.

9. Shi F, Wang J, Shi J, Wu Z, Wang Q, Tang $Z$, et al. Review of Artificial Intelligence Techniques in Imaging Data Acquisition, Segmentation and Diagnosis for COVID-19. IEEE Reviews in Biomedical Engineering. 2020. doi:
10.1109/RBME.2020.2987975.

10.Pourhomayoun M, Shakibi M. Predicting Mortality Risk in Patients with COVID-19 Using Artificial Intelligence to Help Medical Decision-Making. MedRxiv. 2020. doi: 10.1101/2020.03.30.20047308.

11.Guyon I, Elisseeff A. An introduction to variable and feature selection. Journal of machine learning research. 2003;3:1157-82. 